\title{
Utilization of stimulated Raman Scattering as secondary pump on hybrid remotely- pump L-band Raman/erbium-doped fiber amplifier.
}

\begin{abstract}
An L-band remotely-pumped erbium-doped fiber amplifier incorporating a secondary pumping scheme utilizing stimulated Raman Scattering (SRS) was demonstrated. $1423 \mathrm{~nm}$ Raman laser was employed to generate SRS which became the secondary pump source. The amplifier displayed excellent gain of up to $27.3 \mathrm{~dB}$ at $1570 \mathrm{~nm}$ for $-30 \mathrm{dBm}$ input. Noise figures were also kept to a minimum, with the highest figure measured at $11 \mathrm{~dB}$ which was influenced by imperfection of the $\mathrm{C} / \mathrm{L}$ coupler utilized in this architecture. Overall transmission performance was measured as well and demonstrated an encouraging outcome with gain as high as $24 \mathrm{~dB}$ while the noise figure was maintained at about $11 \mathrm{~dB}$. The L-band signal amplification was also contributed by the stimulated Raman scattering along the transmission fiber. The outcome of this study emphasized the feasibility of secondary pumping scheme using SRS in L-band gain enhancement.
\end{abstract}

Item Type: Article 